\title{
ESTRUCTURA LÉXICO-GRAMATICAL DE TÍTULOS DE ARTÍCULOS DE INVESTIGACIÓN DE ODONTOLOGÍA EN ESPAÑOL
}

\author{
LEXICAL GRAMMATICAL STRUCTURE OF DENTISTRY \\ RESEARCH ARTICLE TITLES WRITTEN IN SPANISH
}

\author{
OSCAR ALBERTO MORALES \\ Universidad de Los Andes, Venezuela \\ oscarula@ula.ve \\ BEXI PERDOMO \\ Toulouse Lautrec \\ bexi.de.flores@gmail.com \\ DANIEL CASSANY \\ Universitat Pompeu Fabra \\ daniel.cassany@upf.edu \\ JOEL ACEVEDO \\ Universidad de Los Andes, Venezuela \\ bexi@ula.ve \\ JHON ÁLVAREZ AHLGREN \\ Uppsala University, Suecia \\ jhon.alvarez.ahlgren@ki.se
}

\section{RESUMEN}

Este artículo persigue analizar la estructura sintáctica y la función retórica de 250 títulos de artículos de investigación odontológicos en español. Se examinó cualitativa y cuantitativamente la longitud, la puntuación, la estructura léxico-gramatical y los tipos de títulos. Se encontró un promedio de 15,5 palabras por título. Se identificaron tres tipos de títulos: nominales $(78 \%)$, compuestos $(21,2 \%)$ y oraciones completas $(0,8 \%)$. Predominan los títulos sin puntuación. El 21,2\% usa los dos puntos y el punto y seguido para formar títulos compuestos. Se observaron dos combinaciones retóricas en los títulos compuestos: tema-método y tema-descripción. Predominan las construcciones nominales de un núcleo (nombres no disciplinares) con frases preposicionales (100\%), 
adjetivos (29\%), frases verbales no personales (28\%) y cláusulas relativas (4,4\%) como posmodificadores.

Palabras clave: Análisis léxico gramatical, artículo de investigación, escritura académica, odontología, títulos académicos.

\begin{abstract}
This paper aims to analyze the syntactic structure and rhetorical function of 250 dentistry research articles titles in Spanish. Length, punctuation, lexical-grammatical structure, and styles of titles were examined qualitatively and quantitatively. It was found an average of 15.5 words per title; three types of titles: nominal (78\%), compound $(21.2 \%)$ and full-sentence titles $(0.8 \%)$. Titles without punctuation predominated. $21.2 \%$ used colon and period to form compound titles predominated. It was observed that compound titles combine two rhetorical components: topic-method and topic-description. Uni-head nominal constructions (using mainly non-discipline-specific nouns) using prepositional phrases (100\%), adjectives (29\%), non-personal verbal phrases $(28 \%)$ and relative clauses $(4.4 \%)$ as postmodifiers predominated.
\end{abstract}

Keywords: Academic titles, academic writing, dentistry, lexical grammatical structure, research paper.

Recibido: 20/07/2020. Aceptado:18/11/2020.

\title{
1. INTRODUCCIÓN
}

- $\mathrm{n}$ las últimas tres décadas, los estudios discursivos de géneros académicos y publicaciones científicas han analizado mayormente la estructura del artículo de investigación (AI, en adelante) publicado en inglés (Afful, 2017; Ye, 2019) y las secciones que los constituyen (Hopkins y Dudley-Evans, 1988) empleando el modelo de Swales $(1990 ; 2004)$, con el propósito de aplicar los resultados a la enseñanza de la escritura, para desarrollar la conciencia discursiva de los estudiantes.

Salvo escasas excepciones, el AI es el medio de comunicación por excelencia de la comunidad científica internacional; es el género discursivo más prestigioso, ya que por medio de este se disemina y legitima el conocimiento científico en las distintas disciplinas (Swales, 1990; 2004), académicos e investigadores someten a consideración los resultados de sus trabajos entre los científicos de su comunidad, quienes los aceptan como aportación (Swales, 1990).

En odontología, el estudio del discurso académico en español es muy escaso. En el ámbito internacional, destacan los estudios de tesis y artículos científicos en inglés. Karapetjana (2016) estudió la estructura retórica del AI publicados en 
la British Dental Journal. Kostenko (2017) analizó algunos aspectos pragmáticos de casos clínicos (CC, en adelante) publicados en inglés en revistas odontológicas de alto impacto. También se han publicado estudios contrastivos de la sección introducción en persa e inglés (Farnia y Rahimi, 2017), la sección discusión en árabe e inglés (Alharbi, 2016), la estructura de la discusión de AI odontológicos en inglés, tomando como referencia los hallazgos de estudios previos en AI de lingüística aplicada (Basturkmen, 2012), el género abstract en tailandés e inglés (Vathanalaoha y Tangkiengsirisin, 2018) y en español e inglés (Morales, Cassany y Díaz, 2015) y el uso de atenuantes e intensificadores en informes de investigación escritos por estudiantes y profesionales de la odontología (Crosthwaite, Cheung y Jiang, 2017).

En español se ha analizado la estructura retórica y el uso de atenuantes en AI, CC y artículos de revisión tradicionales (Morales, 2010), la estructura retórica de revisiones sistemáticas (RS, en adelante) en español (Morales et al., 2020d), la estructura retórica de tesis (Morales et al., 2020c). Recientemente, los títulos académicos también han comenzado a estudiarse en esta disciplina.

El título se define como una etiqueta descriptiva y concisa del texto, que lo individualiza, resume su contenido y atrae la atención de la audiencia (Soler, 2011). El título es el primer encuentro del lector con un documento (Moattarian y Alibabaee, 2015; Cheng, Kuo y Kuo, 2012; Jalilifar, 2010). Para Jalilifar (2010) es un indicio de identidad del trabajo, que busca espacio en la comunidad discursiva. Salager-Meyer, Alcaraz-Ariza y Luzardo (2013), Haggan (2004) y Jalilifar (2010) consideran que ahorra tiempo y facilita la tarea a lectores y editores. Por ello, se recomienda escribir títulos claros y precisos, que informen sobre el tema del texto, el diseño y el alcance de la investigación (Swales y Feak, 2012).

Recientemente, gracias al auge de la publicación en línea, los académicos tienden a revisar la literatura en bases de datos especializadas y multidisciplinarias. Suelen leer primero el título de los trabajos y, a partir de este, deciden si vale o no la pena leer el texto completo (Jalilifar, 2010; Moattarian y Alibabaee, 2015). Además, hay evidencia de que en ocasiones los académicos en ciencias médicas toman decisiones clínicas a partir de la información que aporta el título de ensayos clínicos (Entralgo, Salager-Meyer y Luzardo, 2015; Salager-Meyer y AlcarazAriza, 2013).

Por otro lado, especialistas en información y documentación catalogan los textos (artículos, libros o tesis) en bases de datos, bibliotecas digitales, directorios y repositorios a partir de los datos que aportan los títulos. Por ello, la revisión de la literatura sobre títulos académicos sugiere que deben informar con precisión sobre el tema y el diseño de la investigación realizada (Busch-Lauer, 2000; Haggan, 2004; Jalilifar, 2010; Swales y Feak, 2012).

Los títulos se han estudiado desde varias perspectivas. Hay trabajos contrastivos que comparan títulos de dos o más géneros discursivos, disciplinas y lenguas 
(Busch-Lauer, 2000; Jalilifar, 2010; soler, 2007; Soler, 2009; Soler, 2011; AlcarazAriza y Salager-Meyer, 2013); otros estudian de forma individual estas variables (Anthony, 2001; Cianflone, 2010; Cianflone, 2012; Cheng et al., 2012; SalagerMeyer y Alcaraz-Ariza, 2013; Soler, 2018). En el campo de la odontología, se encontraron solo tres trabajos de títulos en: RS (Morales et al., 2020a), tesis (Morales et al., 2020b) y un artículo que compara títulos de AI de odontología, lingüística aplicada e ingeniería civil en inglés (Moattarian y Alibabaee, 2015). Hasta la fecha, los títulos de AI de odontología en español no han sido estudiados, por lo que, para llenar este vacío, este trabajo analiza la estructura sintáctica y la función retórica de títulos de AI de odontología en español (2006-2020).

\section{MARCO TEÓRICO}

\subsection{Sobres los títulos académicos}

Para Soler (2011), los títulos son etiquetas que describen y resumen de forma concisa el contenido de un trabajo y atraen la atención de potenciales lectores. Por lo tanto, deben ser atractivos, claros y precisos (Salager-Meyer y Alcaraz-Ariza, 2013). Swales y Feak (2012) proponen varios criterios que debe cumplir un título apropiado de trabajos de investigación (artículos y tesis): indicar el tema y el alcance del estudio, ser autoexplicativo y, en algunos casos, indicar el tipo de estudio. La inclusión de estos datos contribuye a acotar el tema y a seleccionar a su audiencia apropiada (Jalilifar, 2010). A continuación, se resumen varios trabajos sobre algunos aspectos gramaticales y retóricos de los títulos de textos académicos.

\section{a) Longitud}

Se calcula contando el total de palabras que contiene o por cadenas de letras separadas por espacios en blanco o signos de puntuación (Salager-Meyer, Alcaraz-Ariza y Luzardo, 2017; Moattarian y Alibabaee, 2015). Estos autores han considerado las abreviaturas y las palabras compuestas, con o sin guion, como palabras.

Como muestra la Tabla I, hay diferencias culturales, disciplinares y de género en cuanto a la longitud de los títulos (Busch-Lauer, 2000; Anthony, 2001; Swales y Feak, 2012; Moattarian y Alibabaee, 2015). Los hallazgos de Soler (2007) y Nagano (2015) muestran que los títulos de AI en ciencias médicas son más largos que en ciencias sociales. También, los títulos de tesis, tanto en español como en inglés, tienden a ser más largos (Morales et al., 2020b). Similarmente, para Lewison y Hartley (2005), los títulos de AI de ciencia y medicina han ido aumentando con el tiempo. Otros investigadores relacionan la longitud del título con la frecuencia en que se consulta y cita el artículo correspondiente. Así, Paiva, Lima y Paiva (2012) hallan que cuanto más corto e informativo es el título, más consultas y citas recibe. 
Tabla I. Longitud del título en palabras por disciplina y género.

\begin{tabular}{|c|c|c|c|}
\hline Autores & Disciplinas & Géneros & $\begin{array}{l}\text { Promedio en } \\
\text { palabras }\end{array}$ \\
\hline Budgell (s/i) & Medicina & AI & 16 \\
\hline Anthony (2001) & Computación & AI & 8 \\
\hline Haggan (2004) & $\begin{array}{l}\text { Ciencia, literatura y } \\
\text { lingüística }\end{array}$ & AI & 10 \\
\hline Levison y Hartley (2005) & Medicina & AI & 10 \\
\hline Gesuato (2009) & Lingüística & Tesis & 13 \\
\hline \multirow[t]{2}{*}{ Jalilifar (2010) } & \multirow[t]{2}{*}{ Lingüística } & AI & 11 \\
\hline & & Tesis & 14 \\
\hline \multirow[t]{2}{*}{ Afful y Akoto (2010) } & Literatura & \multirow{2}{*}{ Tesis } & 13 \\
\hline & Química & & 10 \\
\hline \multirow[t]{3}{*}{ Morales (2010) } & \multirow[t]{3}{*}{ Odontología } & $\mathrm{CC}$ & 10 \\
\hline & & $\mathrm{AR}$ & 9 \\
\hline & & $\mathrm{AI}$ & 13 \\
\hline \multirow{2}{*}{ Soler $(2007 ; 2009 ; 2011)$} & \multirow{2}{*}{$\begin{array}{l}\text { Ciencias naturales } \\
\text { y ciencias sociales }\end{array}$} & $\mathrm{AI}$ & 14 \\
\hline & & $\mathrm{AR}$ & 9 \\
\hline Salager-Meyer et al. (2013) & Medicina & $\mathrm{CC}$ & 10 \\
\hline \multirow[t]{4}{*}{ Cianflone $(2010 ; 2012 ; 2013)$} & \multirow[t]{2}{*}{ Veterinaria } & AI & 14 \\
\hline & & Carteles & 12 \\
\hline & \multirow{2}{*}{$\begin{array}{l}\text { Tecnología de ali- } \\
\text { mentos }\end{array}$} & Ponencias & 15 \\
\hline & & $\mathrm{AI}$ & 15 \\
\hline \multirow[t]{2}{*}{ Moattarian y Alibabaee (2015) } & $\begin{array}{l}\text { Lingüística aplica- } \\
\text { da, ingeniería civil }\end{array}$ & \multirow[t]{2}{*}{ AI } & 13 \\
\hline & Odontología & & 10 \\
\hline Afful (2017) & Lingüística aplicada & Conferencias & 10 \\
\hline \multirow[t]{3}{*}{ Salager-Meyer et al. (2017) } & \multirow{3}{*}{$\begin{array}{l}\text { Medicina alternati- } \\
\text { va y complementa- } \\
\text { ria (MAC) }\end{array}$} & AI & 14 \\
\hline & & $\mathrm{AR}$ & 13 \\
\hline & & $\mathrm{CC}$ & 14 \\
\hline Shahidipour y Alibabaee (2017) & $\begin{array}{l}\text { Ingeniería, psiquia- } \\
\text { tría y lingüística }\end{array}$ & AI & 13 \\
\hline Morales et al. (2020a) & Odontología & RS & 14 \\
\hline Morales et al. (2020b) & Odontología & Tesis en inglés & 13 \\
\hline
\end{tabular}




\section{b) Estructura sintáctica}

Siguiendo estudios previos, se distinguen tres tipos:

- Oraciones completas. Los títulos con estructura de frase con un verbo conjugado (Haggan, 2004; Soler, 2007; Soler, 2009; Soler, 2011; Cheng et al., 2012). Suelen adelantar los resultados del estudio (Jalilifar, 2010; Cianflone, 2012; Cianflone, 2013). También se pueden presentar en forma de pregunta (Nagano, 2015).

- Títulos compuestos. Están constituidos por dos o más componentes o unidades, las cuales pueden adoptar diferentes estructuras gramaticales (frases nominales, interrogativas, verbales no personales, adverbiales, proposicionales y gerundios) y se yuxtaponen con un signo de puntuación (Jalilifar, 2010; Cianflone, 2012; Cianflone, 2013; Alcaraz-Ariza y Salager-Meyer, 2013; Entralgo et al., 2015).

- Sintagmas. Son títulos de una sola unidad. Se subdividen en tres tipos: frases nominales, preposicionales y verbales no personales, con participio, gerundio o infinitivo (Jalilifar, 2010; Cianflone, 2012; Cianflone, 2013; Alcaraz-Ariza y Salager-Meyer, 2013; Entralgo et al., 2015).

\section{c) Relaciones retóricas de los componentes}

Swales y Feak (2012) sugieren cuatro relaciones posibles entre los elementos de un título compuesto: problema-solución, general-específico, tema-método y mayormenor. Por su parte, Anthony (2001) identificó otras relaciones en títulos de AI en informática: nombre-descripción, descripción-nombre, tema-alcance, temamétodo y tema-descripción. Coincidiendo con estos autores, la Tabla II resume las combinaciones halladas en títulos de RS y tesis odontológicos.

Tabla II. Combinaciones retóricas observadas en títulos en odontología.

\begin{tabular}{|l|c|c|}
\hline \multicolumn{1}{|c|}{ Género } & Idioma & Combinaciones \\
\hline RS (Morales et al., 2020a) & Español & $\begin{array}{c}\text { Tema-método } \\
\text { Pregunta-método } \\
\text { Tema-descripción-método } \\
\text { Tema-alcance-método }\end{array}$ \\
\hline Tesis (Morales et al., 2020b) & Inglés & $\begin{array}{c}\text { Tema-método } \\
\text { Tema-alcance } \\
\end{array}$ \\
& & Pregunta-tema \\
& Pregunta-método \\
\hline
\end{tabular}




\section{d) Análisis semántico}

Los títulos sintetizan el contenido de una investigación. Por lo tanto, se han analizado con varios indicadores semánticos: la densidad semántica, la frecuencia y el grado de especificidad de la terminología (Gesuato, 2009), la densidad léxica (Afful y Akoto, 2010), el uso de nombres disciplinares y no disciplinares (Wang y Bai, 2007; Cheng et al., 2012; Rath, 2010a; Rath, 2010b; Rath, 2010c), las nominalizaciones (Rath, 2010c) y la informatividad (Jalilifar, 2010; Rath, 2010a; Rath, 2010b; Rath, 2010c).

\section{METODOLOGÍA}

En este estudio se adopta el enfoque de múltiples perspectivas (Applied genre analysis; Bhatia, 2002). Integra aportes de la "Escuela de la Nueva Retórica Estadounidense", sobre todo el papel relevante del contexto (Berkenkotter y Huckin, 1995), al modelo swalesiano de análisis de género (Swales, 1990; Swales, 2004).

Por ello, se combinan el análisis textual del corpus de estudio y el análisis contextual. Mediante el análisis textual se examina manualmente el corpus: revisores independientes (lingüistas y odontólogos) leen varias veces el corpus para caracterizarlo retóricamente e identificar las estructuras y sus funciones dentro del título. Como parte del análisis contextual se realizan entrevistas a odontólogos considerados informantes expertos, miembros reputados de la comunidad odontológica (profesores, investigadores y autores), para explorar su interpretación en relación con la estructura de los títulos de AI.

\subsection{Corpus}

Se seleccionó una muestra aleatoria de 250 títulos de AI odontológicos escritos en español, publicados en tres revistas entre 2008 y 2020: Revista Clínica de Periodoncia, Implantología y Rehabilitación Oral, Revista Odontológica Mexicana y Revista Española de Cirugía Oral y Maxilofacial. Estas revistas están indexadas en Medline, Scopus, SciELO, Dialnet y Latindex. En total, el corpus suma 3861 palabras, con un promedio de 15,5 palabras por título.

Los AI se descargaron de la base de datos Elsevier (vía Science Direct) en el área medicina y odontología, subespecialidad odontología, con el filtro "Artículo de investigación". Como algunos CC y RS se catalogan erróneamente como AI, se revisaron los textos completos, con la asesoría de los informantes, para verificar que fueran $\mathrm{AI}$ auténticos. Los autores de los $\mathrm{AI}$ son hispanohablantes (1L o 2L), provenientes de 10 países latinoamericanos y de España. El 96\% de los AI tienen coautoría, con un promedio de 4 autores por artículo, oscilando entre 1 y 13 au- 
tores. Todos los autores están adscritos a universidades, centros de investigación $\mathrm{u}$ hospitales universitarios, y tienen estudios de postgrado (doctorado, maestría o especialidad).

El número de títulos analizados se consideró suficiente para el propósito planteado. Trabajos previos han empleado corpus de tamaños similares o más pequeños (Soler, 2009; Salager-Meyer y Alcaraz-Ariza, 2013; Moattarian y Alibabaee, 2015; Entralgo et al., 2015; Morales et al., 2020a). Además, algunos autores han resaltado los beneficios de hacer estudios discursivos con muestras relativamente pequeñas (Banks, 2005; Belcher, 2005).

\subsection{Criterios de análisis}

El corpus fue analizado de modo cualitativo y cuantitativo a partir de los aspectos tratados en trabajos previos (Anthony, 2001; Moattarian y Alibabaee, 2015):

- Puntuación: qué signos se usan y con qué propósito.

- Extensión: número de palabras según el contador automático de MS Word® y según el recuento manual.

- Estilos: títulos de una unidad y su tipo (oración completa, frase nominal, preposicional o verbales no personal) y títulos compuestos.

- Estructuras morfosintácticas: estructura sintáctica y elementos constitutivos de los títulos y sus componentes (frases nominales, verbales, preposicionales y cláusulas relativas). También se identificaron sustantivos, construcciones verbales, personales y no personales, adjetivos, artículos y preposiciones usadas como modificadores.

- Relaciones retóricas: relación retórica de los componentes de los títulos compuestos.

- Densidad léxica: porcentaje de palabras léxicas y gramaticales.

- Términos disciplinares y metodológicos: presencia o no de términos sobre metodología de la investigación y sobre la disciplina.

\section{RESULTADOS}

\subsection{Uso de puntuación}

Predominan los títulos sin signos de puntuación $(\mathrm{n}=197)$. Este rasgo se asocia a la estructura sintáctica predominante: títulos de una unidad en forma de título nominal (ver más adelante), lo cual es una característica de los títulos de distintos géneros en una variedad de disciplinas y lenguas en AI y AR (Jalilifar, 2010; Shahi- 
dipour y Alibabaee, 2017; Busch-Lauer, 2000; Haggan, 2004; Soler, 2007; Soler, 2009; Soler, 2011; Cheng et al., 2012; Nagano, 2015) en CC (Salager-Meyer y Alcaraz-Ariza, 2013; Salager-Meyer et al., 2013; Salager-Meyer et al., 2017) y en tesis en inglés y español (Dudley-Evans, 1984; Jalilifar, 2010; Soler, 2018; Morales et al., 2020b). No obstante, estos resultados difieren del uso de la puntuación en RS en odontología, en los que son más frecuentes los títulos con punto y seguido o dos puntos (Morales et al., 2020a).

La Tabla III muestra que el 21,2\% usa puntuación para separar los componentes de los títulos compuestos: dos puntos $(11,6 \%)$, punto y seguido $(7,6 \%)$, coma $(1,6 \%)$ y punto y coma $(0,4 \%)$.

Tabla III. Frecuencia de uso de signos de puntuación.

\begin{tabular}{|c|r|c|}
\hline Punctuation & fi & $\%$ \\
\hline$\varnothing$ & 197 & 78,8 \\
\hline Punto y seguido & 19 & 7,6 \\
\hline Dos puntos & 29 & 11,6 \\
\hline Coma & 4 & 1,6 \\
\hline Punto y coma & 1 & 0,4 \\
\hline Total & 250 & 100 \\
\hline
\end{tabular}

El uso predominante de punto y seguido y dos puntos coincide con estudios previos sobre títulos de CC médicos en inglés (Salager-Meyer et al., 2013), AI (Lewison y Hartley, 2005), tesis de lingüística en inglés (Jalilifar, 2010; Afful y Akoto, 2010; Haggan, 2004), RS odontológicas en español (Morales et al., 2020a) y AI de lingüística y literatura en inglés (Haggan, 2004). Es decir, estos signos de puntuación parecen ser los más utilizados en títulos compuestos en varias disciplinas.

Los ejemplos (1-4) ilustran el uso de la puntuación para formar títulos compuestos: (1) muestra un ejemplo de los dos puntos; el ejemplo (2), el uso del punto y seguido; el (3), la coma; y el único caso de punto y coma se muestra en el ejemplo (4).

(1) Manejo de la osteonecrosis maxilar asociada al uso de medicamentos en virtud de su estadio clínico: análisis de 19 casos

(2) Cambios en la dimensión de la vía aérea en pacientes con secuencia de PierreRobin asociada a síndromes malformativos tras distracción mandibular. Planificación del vector 
(3) Capacidad buffer de la saliva en presencia de bebidas energéticas comercializadas en Chile, estudio in vitro

(4) Relación entre la oclusión traumática y abfracciones; su rol en las afecciones pulpares

\subsection{Longitud}

Se halló un promedio de 15,5 palabras por título (con un rango entre cinco y 38 palabras). Esta media es superior a los promedios observados en los estudios en distintos géneros discursivos referidos en la Tabla I, excepto el trabajo de Cianflone (2013) sobre títulos de ponencias y AI en tecnología de los alimentos en inglés (promedio de 15 palabras). Esto indica que los títulos de AI odontológicos en español suelen ser relativamente largos; superan por lo general las 12 palabras establecidas en los "Requisitos de uniformidad para manuscritos enviados a revistas biomédicas", conocidos como Estilo Vancouver. Estas normas rigen redacción y preparación de artículos en las revistas de donde se obtuvo el corpus (Comité Internacional de Editores de Revistas Médicas, 2006).

La Tabla IV muestra el rango de extensión de los títulos. La mayoría se ubica entre 10 y 20 palabras (73,2\%). Son pocos frecuentes los títulos muy cortos (menos de 10 palabras) y los muy largos (más de 20 palabras).

Tabla IV. Frecuencia de la extensión de los títulos.

\begin{tabular}{|l|c|c|}
\hline \multicolumn{1}{|c|}{ Rango } & Frecuencia & $\%$ \\
\hline$\leq 9$ & 30 & 12 \\
\hline $10-15$ & 109 & 43,6 \\
\hline $16-20$ & 74 & 29,6 \\
\hline $21-30$ & 33 & 13,2 \\
\hline$\geq 31$ & 4 & 1,6 \\
\hline Total & 250 & 100 \\
\hline
\end{tabular}

Estos datos apuntan una preferencia por los títulos largos en los AI odontológicos, contradiciendo lo hallado en otras disciplinas que consideran la brevedad como un elemento clave en los títulos (Soler, 2007; Jalilifar, 2010). Es factible que este resultado se deba, por un lado, a que la Revista Española de Cirugía Oral y Maxilofacial no regula la extensión del título. En los otros casos, algunos autores incumplen las instrucciones referidas al título, las cuales establecen que la extensión de los títulos no debe superar los 100 caracteres (aproximadamente 20 
palabras), que el $20 \%$ de los títulos supera. Por su parte, la Revista Odontológica Mexicana limita la extensión a 14 palabras. Sin embargo, 50\% de los títulos superan ese límite.

(5) Estomatotoxicidad bucal inducida por quimioterapia [5 palabras]

(6) Estudio comparativo de la resistencia al desprendimiento de tubos adheridos a una superficie de resina obturada sobre la superficie bucal de los molares con la resina Empress Direct y con la resina Transbond XT: un estudio ex vivo [38 palabras]

El ejemplo (5) es el título más corto y el (6), el más largo del corpus. En ambos casos se viola la máxima de cantidad de Grice (1975). En (5) se aporta menos información de la requerida; no se especifica el tipo de cáncer, el perfil de los pacientes o el tipo de tratamiento quimioterapéutico. En (6) hay redundancia y repeticiones innecesarias. Por lo tanto, ambos títulos son inapropiados.

\subsection{Estilos de títulos}

La Tabla V muestra el predominio de títulos nominales de una unidad, seguido de los compuestos. Solo se reportaron dos títulos en forma de oración completa. Esta tendencia coincide con numerosos estudios de varios géneros en distintas disciplinas y lenguas: Jalilifar (2010), Shahidipour y Alibabaee (2017), BuschLauer (2000), Haggan (2004), Soler (2007; 2009; 2011), Cheng et al. (2012) y Nagano (2015) en AI y AR en inglés; Salager-Meyer y Alcaraz-Ariza (2013), Salager-Meyer et al. $(2013 ; 2017)$ en CC médicos en inglés; y Dudley-Evans (1984), Jalilifar (2010), Soler (2018) y Morales et al. (2020b) en tesis en español e inglés. Por otro lado, la baja frecuencia de títulos de oraciones completas coincide con Haggan (2004) y Wang y Bai (2007), quienes encontraron proporciones similares en AI en inglés. En cambio, este resultado difiere de otros trabajos de AI y RS, que muestran una frecuencia más alta de títulos compuestos (Haggan, 2004; Soler, 2007; Morales et al., 2020a).

Tabla V. Estilos de títulos.

\begin{tabular}{|c|c|c|}
\hline Tipo de título & Núm. de títulos & $\%$ \\
\hline Nominales & 195 & 78 \\
\hline Compuestos & 53 & 21,2 \\
\hline Oración completa & 2 & 0,8 \\
\hline Total & $\mathbf{2 5 0}$ & $\mathbf{1 0 0}$ \\
\hline
\end{tabular}




\subsubsection{Títulos nominales}

El 78\% son títulos nominales. Esta alta frecuencia coincide con estudios previos en AI que hallaron frecuencias por encima del 90\% (Busch-Lauer, 2000; Haggan, 2004; Soler, 2007; Jalilifar, 2010; Cheng et al., 2012; Salager-Meyer y AlcarazAriza, 2013; Nagano, 2015; Shahidipour y Alibabaee, 2017). También coincide con los resultados de títulos de tesis, entre los cuales destacan Dudley-Evans (1984), Jalilifar (2010), Soler (2018) y Morales et al. (2020b); pero es mayor que los de Gesuato (2009) en tesis de lingüística (51\%).

Se encontró títulos de un núcleo nominal $(92,4 \%)$ y de dos núcleos $(7,6 \%)$. El predominio de los títulos nominales de un solo núcleo coincide con estudios en artículos y tesis (Wang y Bai, 2007; Jalilifar, 2010). Predominan los núcleos nominales no disciplinares (57,2\%); el otro 46,8\% emplea nombres disciplinares. Rath (2010a; 2010b; 2010c) y Cheng et al. (2012) también identificaron estos tipos de núcleos nominales, siendo más frecuentes los no disciplinares. Entre estos últimos, predomina la identificación de la investigación mediante el uso de términos abstractos. En los ejemplos (7 y 8 ) se muestran títulos de uno y dos núcleos, que emplean nombres disciplinares. En cambio, (9) y (10) son títulos de un núcleo que emplean términos no disciplinares.

(7) Cuidado bucal en mayores dependientes de un programa de cuidados domiciliarios

(8) Flujo salival y caries radicular en adultos mayores autovalentes

(9) Eficacia del uso del plasma rico en factores de crecimiento en defectos periodontales distales de segundos molares inferiores, posterior a la extracción de un tercer molar mandibular

(10) Estudio de confiabilidad de la prueba de sialometría para flujo no estimulado en sujetos adultos clínicamente sanos

En los títulos nominales, el significado del núcleo a menudo se amplía con posmodificadores que aportan datos específicos adicionales. Esta estructura es característica de los títulos de géneros académicos (Soler, 2007); tiene la función de sintetizar la información con brevedad y presentar una sinopsis del contenido y el diseño de estudio (Rath, 2010c). Todos los títulos, tanto nominales como compuestos, incluyeron posmodificadores, predominantemente frases preposicionales (100\%), seguidas de adjetivos (29\%), frases verbales no personales, participios, infinitivos y gerundios (28\%), y cláusulas subordinadas (4,4\%). Morales et al. (2020b) encontraron resultados similares en tesis odontológicas en inglés.

Los ejemplos (11-13) combinan el uso de adjetivos con frases preposicionales para caracterizar el tema y el tipo de estudio. En (13) se observa el uso del participio "asociados" para especificar el tipo de microrganismos analizado. Finalmente, 
(14) incluye una cláusula subordinada adjetiva para caracterizar al paciente.

(11) Análisis químico elemental y de fases por medio de PIXE, DSC, TGA y DRX en MTA Angelus ${ }^{\circledR}$ y un cemento Portland blanco

(12) Estudio Comparativo de la Especificidad Dimensional del Cone Beam y la Radiografía Panorámica Digital

(13) Caries dental y microorganismos asociados a la caries en la saliva de los alumnos del primer año de la Facultad de Odontología, UNAM

(14) Caracterización del paciente que solicita atención de especialidad en periodoncia en una población chilena

La tendencia a usar posmodificadores puede reflejar el estilo comprimido del discurso académico en odontología. Este se caracteriza por usar abundantes frases preposicionales en lugar de oraciones completas y cláusulas verbales dependientes en la construcción discursiva del título. Este resultado es consistente con los hallazgos de Biber y Gray (2010), Jalilifar (2010), Cheng et al. (2012), Moattarian y Alibabaee (2015) y Shahidipour y Alibabaee (2017), Wang y Bai (2007) y Gesuato (2009), quienes hallaron que las frases preposicionales son las estructuras más utilizadas como posmodificadores en los títulos nominales, seguidas de las frases verbales no personales.

Así mismo, se encontró un promedio de cuatro preposiciones por título, encabezando frases que funcionan como posmodificadores, lo cual coincide con Afful y Akoto (2010) y Morales et al. (2020b). Estas expresiones comprimidas son más económicas y sintéticas, y, como señalan Biber y Gray (2010), facilitan una lectura rápida y eficiente. Haggan (2004) considera que este es un rasgo distintivo de la escritura académica.

Por otro lado, la frecuencia de uso de premodificadores es significativamente más baja. Solo el $2 \%$ de los títulos nominales incluye algún tipo de premodificador, lo cual coincide con los hallazgos de Morales et al. (2020a). Como lo ha sugerido Soler (2011), esta estructura sintáctica es menos usada en castellano que en inglés. En este corpus, solo se encontraron artículos determinantes tanto en singular como en plural, como se muestra en el ejemplo (15).

(15) La asociación entre el arco de sonrisa y los corredores bucales con el biotipo facial en sujetos con normoclusión

\subsubsection{Titulos compuestos}

El 21,2\% de los títulos son compuestos constituidos por dos unidades $(\mathrm{n}=53)$. Ambos componentes son frases nominales modificadas por adjetivos, grupos preposicionales o frases verbales no personales. Esta frecuencia es significativamente 
menor que la hallada en trabajos sobre títulos de tesis: Jalilifar (2010) en lingüística aplicada (21,16\%), Afful y Akoto (2010) en literatura (31\%) y Gesuato (2009) en lingüística (48\%). También es menor que en RS odontológicas en español (90\%) en Morales et al. (2020a); pero es mayor que en títulos de tesis en química (3\%), en Afful y Akoto (2010).

Los siguientes ejemplos son prototípicos. El (16) combina el tema con el método. En el (17), el primer componente indica el tema y el segundo, su descripción. Morales et al. (2020a) también encontraron una frecuencia alta del tipo de estructura que ejemplifica (16) en RS odontológicas en español.

(16) Amoxicilina para prevenir la infección postexodoncia de terceros molares incluidos: ensayo clínico aleatorizado

(17) Edentulismo en adultos de Pachuca, México: aspectos sociodemográficos y socioeconómicos

\section{- Combinaciones retóricas de los componentes}

En los 53 títulos compuestos, hay sólo dos combinaciones retóricas: tema-método ( $\mathrm{n}=19)$, como en el ejemplo (16) y tema-descripción, como en el ejemplo (17). En el primer componente se incluye el tema (patología, tratamiento, material, efecto, efectividad, asociación objeto de estudio), en el segundo se incluye la identificación del método u otros detalles de interés. Esta tendencia es similar a los resultados de Moattarian y Alibabaee (2015), Shahidipour y Alibabaee (2017) y Morales et al. (2020a). Rath (2010a), en cambio, encontró que se usa en ambos componentes.

Contrario a lo que indican las declaraciones CONSORT para ensayos clínicos (Schulz, Altman y Moher, 2010) y STROBE para estudios observacionales (Von Elm et al., 2014), la mayoría ( $\mathrm{n}=197)$ no identifica el tipo de estudio en el título. Además, esto difiere de los resultados de Rath (2010a) y Salager-Meyer y AlcarazAriza (2013) y de las recomendaciones de Swales y Feak (2012), quienes sugieren incluir información metodológica del diseño del estudio realizado, mediante títulos que denominan Research procedure titles (Salager-Meyer y Alcaraz-Ariza, 2013).

Estos resultados difieren de los hallazgos de estudios previos en odontología, en los que se muestra que la mayoría identifica el tipo de estudio en el título: Morales (2010) en AI, CC y AR, y Morales et al. (2020a) en RS. Además, contrario a los resultados del presente estudio, Rath (2010a; 2010b; 2010c) sugiere que los títulos de AI suelen ser más efectivos cuando incluyen información metodológica combinada con información del contenido disciplinario específico del texto mediante frases nominales. Esto puede deberse, con base en los testimonios de los informantes expertos, a que no hay necesidad de señalar el diseño cuando se trata de AI, pues es el género central del discurso de la odontología. Pero los "géneros secundarios" (CC, AR y RS) sí deben diferenciarse en el título. Además, el uso de 
nombres no disciplinares, como efecto, efectividad, asociación, prevalencia, in vitro, identifica el diseño de estudio.

En cambio, hay similitudes entre este estudio y el de Morales et al. (2020b), en el que se encontró que la mayoría de las tesis odontológicas en inglés no identifica el tipo de estudio en el título. También son similares a los hallazgos de Jalilifar (2010), quien halló que solo el 8\% de títulos de AI en lingüística aplicada incluyeron información sobre el método.

Entre los términos empleados, se observaron sobre todo ensayo clínico, estudio prospectivo, retrospectivo, comparativo, in vitro, ex vivo, lo cual coincide con las recomendaciones de la declaración CONSORT para ensayos clínicos (Schulz et al., 2010) y la declaración STROBE para estudios observacionales (Von Elm et al., 2014).

\subsubsection{Títulos de oraciones completas}

Se hallaron solo dos títulos en forma de oraciones completas declarativas afirmativas (18). Se suelen considerar títulos conclusivos, pues informan sobre los resultados y las conclusiones del estudio. Estos resultados son consistentes con los de Cheng et al. (2012), Haggan (2004), Jalilifar (2010), Moattarian y Alibabaee (2015), Soler (2007), Shahidipour y Alibabaee (2017) en AI, Morales et al. (2020a) en RS y Afful y Akoto (2010) en tesis, que encontraron poca frecuencia de uso de títulos de oración completa.

(18) La modulación de la expresión de IL-10 por el polimorfismo IL-10-592C/A (rs1800872) es independiente de la presencia y carga bacteriana de los periodontopatógenos clásicos

\subsection{Rasgos semánticos}

En cuanto al tipo de palabra, se encontró un promedio de 10 palabras léxicas y 6 gramaticales por título, lo cual representa una densidad léxica cercana al 64\%. Este resultado es similar a Gesuato (2009), Afful y Akoto (2010), Nagano (2015) y Morales et al. (2020b), quienes encontraron promedios similares en la densidad léxica de títulos de artículos científicos y tesis.

Los nombres no disciplinares, que Rath (2010a) denomina grupos nominales vinculados al proceso de investigación, se usaron con mucha frecuencia $(57 \% ; \mathrm{n}=$ 143). Esto coincide con otros estudios (Rath, 2010a; Rath, 2010b; Rath, 2010c; Cheng et al., 2012;). Rath (2010a) encontró que el $72 \%$ de los títulos incluye este grupo nominal. Cheng et al. (2012) identificaron dos tipos de núcleos nominales, nombres disciplinares y no disciplinares. Entre estos últimos predomina la identi- 
ficación de la investigación mediante el uso de términos abstractos. Rath (2010a; 2010b; 2010c), por su parte, encontró que los nombres no disciplinares, referidos a procesos o al diseño de investigación, que puede incluir una palabra o un grupo nominal, se usan tanto en títulos nominales como en compuestos; en estos últimos puede ubicarse en la primera o en la segunda posición.

La mayoría son nombres abstractos: investigación, estudio, análisis, evaluación, influencia, efectividad, efecto, eficacia, asociación, relación, diseño, propuesta, frecuencia, prevalencia. Sahragard y Meihami (2016), Cheng et al. (2012), Wang y Bai (2007), Jalilifar (2010) y Rath (2010a; 2010b; 2010c) coinciden con estos datos en que este tipo de palabras informan sobre los resultados, la naturaleza o el diseño del estudio. Rath (2010a; 2010b; 2010c) encontró grupos nominales similares en títulos de lingüística aplicada.

Los términos investigación, estudio y análisis suelen ir acompañados del posmodificador comparativo, para indicar que se trata de un trabajo experimental que contrasta varios grupos, como muestra el ejemplo (19). También, los términos: comparación, evaluación, influencia, efectividad, efecto y eficacia, modificados por el tratamiento objeto de estudio, se usan para identificar estudios experimentales y ensayos clínicos (20). En cambio, frecuencia y prevalencia designan estudios epidemiológicos observacionales descriptivos (21). Por su parte, asociación y relación se emplean en estudios observacionales analíticos para establecer relaciones entre dos entidades (casos y controles, estudios de cohorte) (22).

(19) Estudio Comparativo de la Especificidad Dimensional del Cone Beam y la Radiografía Panorámica Digital

(20) Comparación de la precisión marginal de cofias de zirconia entre los sistemas CAD/CAM Cerec InLab (Sirona $\left.{ }^{\circledR}\right)$, CAD/CAM Zirkonzahn (Zirkonzahn®) y sistema pantográfico Zirkograph 025 ECO (Zirkonzahn $\left.{ }^{\circledR}\right)$

(21) Prevalencia de Caries y Pérdida de Dientes en Población de 65 a 74 Años de Santiago, Chile

(22) Asociación entre periodontitis crónica, interleuquina-6 (IL-6) e índice de masa corporal (IMC) en embarazadas

Por otro lado, 57 títulos incluyen nombres compuestos (22,8\%), generalmente referidos a nombres disciplinares, como se muestra en el ejemplo ( 23 y 26). Asimismo, solo el $6 \%$ de los títulos incluyeron nomenclatura de nombres científicos de productos naturales o bacterias (24), y el 10,8\% incluye abreviaturas (25 y 26).

Las nominalizaciones son un rasgo distintivo del discurso científico en general y de los títulos en particular. Permiten expresar mucha información de forma sintética, coherente y cohesiva (Rath, 2010c). En el 92\% de los títulos se usan nominalizaciones, coincidiendo con Rath (2010b). Para este autor, las nominalizaciones ayudan a los escritores a proporcionar más datos en una estructura de 
tipo nominal y también a crear cohesión, como en el ejemplo (26).

En 59 de los títulos $(23,6 \%)$ hay datos sobre el lugar del estudio, y en el 5,2\% $(\mathrm{n}=13)$ sobre el periodo de tiempo incluido en la muestra, predominantemente en estudios epidemiológicos. Como en Jalilifar (2010) y Morales et al. (2020b), los informantes consideran necesario indicarles a los lectores en el título el alcance de la investigación, el contexto social donde se realizó el estudio o la identificación de los individuos participantes, como en los ejemplos (23 y 24).

(23) Análisis histopatológico de casos de cáncer oral en instituciones de las regiones chilenas del Maule y Bío-Bío entre los años 2001-2011

(24) Genotipificación de Porphyromonas gingivalis en Pacientes con Periodontitis

(25) Eficacia de la azitromicina asociada al RAR en periodontitis crónica: ensayo clínico, aleatorizado, controlado y triple ciego en grupos en paralelo

(26) Rehabilitación de una maxila atrófica con el uso de implantes inclinados «Allon-Four»

\section{Informatividad}

Los resultados indican que los títulos de AI en odontología son altamente informativos. Todos los títulos identifican de forma explícita el tema objeto de estudio; establecen el alcance del estudio, el contexto donde se realizó y el área a la que pertenece. Esto coincide con Rath (2010a; 2010b), Jalilifar (2010) y Morales et al. (2020b).

Por su parte, el $80 \%(n=200)$ anuncia resultados con los recursos léxicos señalados en la sección anterior. El $72 \%(\mathrm{n}=180)$ indica el alcance identificando el área, los participantes o el contexto en que se realiza el estudio. Asimismo, el 79,2\% ( $\mathrm{n}=198)$ incluye núcleos nominales que indican acciones, como evaluación, análisis, ensayo, investigación o estudio, lo cual coincide con Rath (2010a; 2010b) y Jalilifar (2010). Según Rath (2010a; 2010b; 2010c) y Cheng et al. (2012), la inclusión aporta información valiosa sobre la naturaleza de la investigación. La alta frecuencia con que se menciona el tema, los resultados y el alcance (100, 80 y $72 \%$, respectivamente) coincide con las indicaciones dadas en estudios previos para estructurar títulos apropiados (Haggan, 2004; Swales y Feak, 2012). Según los informantes expertos, algunos términos no disciplinares, como efectividad, eficacia, asociación, usados como núcleos nominales, permiten adelantar que un estudio experimental evalúa el efecto de una determinada terapia sobre alguna enfermedad, o un estudio observacional analítico muestra la asociación entre dos condiciones.

Finalmente, en el 20,4\% de los títulos se identifica de forma explícita el tipo de estudio. Este bajo porcentaje supone que no se siguen las pautas para informar sobre resultados de investigaciones biomédicas, propuestas en la EQUATOR (Enhancing the QUAlity and Transparency Of health Research) Network (2020), las declaraciones CONSORT para ensayos clínicos (Schulz et al., 2010) y STROBE 
para estudios observacionales (Von Elm et al., 2014). Tampoco coincide con los resultados de Salager-Meyer y Alcaraz-Ariza (2013) y Morales et al. (2020a), que recomiendan incluir datos metodológicos del diseño en el título, ni con Sahragard y Meihami (2016), quienes encontraron que cerca del 50\% de los títulos incluyó información metodológica.

\section{CONCLUSIONES}

Los resultados indican que, a pesar de tener rasgos comunes con títulos de otras disciplinas, la estructura léxico-gramatical de los títulos de AI de odontología en español tienen características particulares. Los títulos tienden a ser más largos que en otras disciplinas y en otros géneros discursivos en varias disciplinas y lenguas. Además, no suelen identificar explícitamente el diseño del estudio. Esto puede deberse a que los autores suelen prestar mayor atención al contenido que a la extensión del título. Además, es un indicador de que no siguen las instrucciones de las revistas, las normas Vancouver ni lo establecido en las declaraciones CONSORT y STROBE referidas a los títulos de AI. Por lo tanto, los estudiantes y odontólogos en formación deberían recibir instrucción formal al respecto.

En cuanto a identificar el diseño del estudio en el título, consideran que es suficiente con la inclusión de términos no disciplinares metodológicos, como efectividad, efecto, eficacia, asociación, análisis comparativo, a partir de los cuales se puede inferir el diseño y el tipo de investigación.

Desde el punto de vista sintáctico, predominan los títulos nominales de una unidad, modificados con frases preposicionales, adjetivos, cláusulas relativas y frases verbales no personales. En cuanto a la estructura de los títulos compuestos, se registraron solo dos (tema-método; tema-descripción), relacionados con dos puntos y punto y seguido. En ambos componentes se emplean construcciones nominales modificadas por adjetivos, frases preposicionales, verbales no personales $y$, en menor medida, cláusulas relativas.

Los resultados indican que los títulos de AI en odontología son altamente informativos, identifican de forma explícita el tema objeto de estudio y establecen el alcance del estudio, el contexto y el área de especialidad. Coherentemente, se encontró una densidad léxica de $64 \%$ y una alta frecuencia de uso de nombres no disciplinares y nominalizaciones.

Estos resultados tienen implicaciones didácticas para todos los involucrados en el desarrollo de la escritura académica: estudiantes, profesores de investigación, odontólogos en formación y docentes de escritura. Como afirma Soler (2007), escribir títulos de textos científicos es una tarea difícil y demandante, ya que los autores requieren haber desarrollado una serie de habilidades y conocimientos que permitan incluir los requisitos que facilitan que los títulos sean apropiados a 
los géneros clave de cada disciplina. Por lo tanto, las habilidades requeridas para escribir buenos títulos de AI de odontología en español se deberían enseñar en el ámbito odontológico universitario tan pronto como sea posible.

La presente investigación muestra evidencia que da cuenta de particularidades de los títulos de AI odontológicos en español. En este sentido, se ratifica, como en Rincón, Narváez y Roldán (2011), la necesidad de formalizar la enseñanza de la investigación y la escritura académica relacionada con los AI, con la participación de odontólogos (insiders) y lingüistas y educadores (outsiders). De esta manera, se puede impulsar la publicación de artículos científicos en revistas de alto impacto, para difundir de manera más efectiva el conocimiento que se produce en nuestras universidades.

Aunque este estudio identifica patrones discursivos en los títulos de AI en odontología, como se proponía, no está exento de limitaciones. El corpus de estudio es pequeño, lo cual limita la posibilidad de ofrecer datos más concluyentes. Además, se aborda un solo género académico, en una disciplina y en una lengua; por ello, es necesario realizar más investigaciones que contrasten los títulos de distintos géneros discursivos que se usan en odontología, los títulos odontológicos con otras disciplinas científicas y los títulos en español con otras lenguas, especialmente el inglés.

\section{Notas}

1. Los ejemplos fueron reproducidos literalmente con su ortografía, tipografía y estructura original.

\section{REFERENCIAS}

Afful, J. (2017). A linguistic analysis of conference titles in applied linguistics. International Journal of Foreign Language Teaching and Research, 5, 18, 11-25. Disponible en: http://jfl.iaun.ac.ir/article_601126_c70f61941e0573535fa204cc7c2322f3. pdf

Afful, J., y Akoto, O. (2010). Cross-disciplinary study of dissertation titles: the case of a University in Ghana. Professional and Academic English, 36, 4-19.

Alcaraz-Ariza, M., y Salager-Meyer, F. (2013). Análisis contrastivo de los títulos en los artículos de investigación de neurología redactados en español e inglés. LSP Journal-Language for Special Purposes, Professional Communication, Knowledge Management and Cognition, 3, 2, 27-50.

Alharbi, S. (2016). Schematic structure of discussion of results sections in the field of Dentistry: A comparison of international and local English-medium journals. Arab World English Journal (AWEJ), 7, 2, 61-76. https://dx.doi. org/10.2139/ssrn.2814814 
Anthony, L. (2001). Characteristic features of research article titles in computer science. IEEE Transactions on Professional Communication, 44, 3, 187-194. https://doi.org/10.1109/47.946464

Banks, D. (2005). The case of Perrin and Thompson: An example of the use of a mini corpus. English for Specific Purposes, 24, 2, 201-213. https://doi. org/10.1016/j.esp.2004.01.001

Basturkmen, H. (2012). A genre-based investigation of discussion sections of research articles in dentistry and disciplinary variation. Journal of English for Academic Purposes, 11, 2, 134-144. https://doi.org/10.1016/j.jeap.2011.10.004

Belcher, D. (2005). Editorial. English for Specific Purposes, 14, 119-121. https:// doi.org/10.1016/j.esp.2004.11.002

Berkenkotter, C., y Huckin, T. (1995). Genre knowledge in disciplinary communication: cognition/culture/power. Nueva York: Lawrence Erlbaum.

Bhatia, V. (2002). Applied genre analysis: a multiperspective model. Ibérica, 4, 3-19.

Biber, D., y Gray, B. (2010). Challenging stereotypes about academic writing: complexity elaboration, explicitness. Journal of English for Academic Purposes, 9, 1, 20-32. https://doi.org/10.1016/j.jeap.2010.01.001

Budgell, B. s/i. Titles of biomedical articles: a corpus-based analysis. Disponible en: https://pdfs.semanticscholar.org/164d/6681f2601a7857c84facd0fd41d77 8783a17.pdf

Busch-Lauer, I. (2000). Titles of English and German research papers in medicine and linguistics. En Anne Trosborg (Ed.), Analysing professional genre. Ámsterdam: John Benjamin, pp. 77-94.

Cheng, S., Kuo, CW. y Kuo, CH. (2012). Research article titles in applied linguistics. Journal of Academic Language and Learning, 6, 1, 1-14. Disponible en: https://journal.aall.org.au/index.php/jall/article/view/178

Cianflone, E. (2010). Scientific titles in veterinary medicine research papers. English for Specific Purposes World, 9, 30, 1-8.

Cianflone, E. (2012). Titles in veterinary medicine research articles. Círculo de Lingüistica Aplicada a la Comunicación, 52, 3-20. http://dx.org/10.5209/rev_ CLAC.2012.v52.41091

Cianflone, E. (2013). Framing research in food science: the state of the art on research article, short communication and poster presentation titles. Revista de Lenguas para Fines Especificos, 19, 269-286. Disponible en: https://accedacris. ulpgc.es/bitstream/10553/12241/1/0233536_00019_0012.pdf

Comité Internacional de Editores de Revistas Médicas. (2006). Requisitos de uniformidad para manuscritos enviados a revistas biomédicas: Redacción y preparación de la edición de una publicación biomédica. Revista Española de Pediatría, 62, 2, 114-136.

Crosthwaite, P., Cheung, L., y Jiang, Kevin. (2017). Writing with attitude: Stance 
expression in learner and professional dentistry research reports. English for Specific Purposes, 46, 107-123. https://doi.org/10.1016/j.esp.2017.02.001

Dudley-Evans, T. (1984). A Preliminary investigation of the writing of dissertation titles. En Gregory James (ed.), The ESP classroom: Methodology, materials, expectations. Londres: Exeter Linguistic Studies, pp. 40-46.

Entralgo, J., Salager-Meyer, F., y Luzardo, M. (2015). ¿Cuán gramaticalmente complejos son los títulos de los artículos científicos en las ciencias naturales? Revista de Lenguas para Fines Especificos, 21, 2, 70-97. Disponible en: https:// accedacris.ulpgc.es/bitstream/10553/15519/1/0233536_00021_0003.pdf

EQUATOR Network. (2020). Disponible en: http://www.equator-network.org (consultado 23-08-2020).

Farnia, M., y Rahimi, S. (2017). Comparative generic analysis of introductions of English and Persian dentistry research articles. Research in English Language Pedagogy, 5, 1, 27-40.

Gesuato, S. (2009). Encoding of information in titles: practices across four genres in linguistics. En Christopher Taylor (ed.), Ecolingua: the role of e-corpora in translation and language learning. Trieste: Ecolingua, pp. 127-157.

Grice, P. (1975). Logic and conversation. En Maite Ezcurdia, \& Robert Stainton (eds.), The semantics-pragmatics boundary in philosophy. Nueva York: Routledge, pp. 41-58.

Haggan, M. (2004). Research paper titles in literature, linguistics and science: dimensions of attraction. Journal of Pragmatics, 36, 2, 293-317. https://doi. org/10.1016/S0378-2166(03)00090-0

Hopkins, A., y Dudley-Evans, T. (1988). A genre-based investigation of the discussion sections in articles and dissertations. English for Specific Purposes, 7, 2, 113-121. https://doi.org/10.1016/0889-4906(88)90029-4

Karapetjana, R. (2016). Rhetorical structure of the research article in dentistry. Russian Linguistic Bulletin, 3, 10-14.

Kostenko, V. (2017). Pragmatic and Communicative Aspects of Case Reports in Dentistry. Tesis doctoral. Poltava, Ucrania: University of Poltava.

Jalilifar, A. (2010). Writing titles in applied linguistics: a comparative study of theses and research articles. Taiwan International ESP Journal, 2, 1, 27-51. http:// dx.doi.org/10.6706\%2fTIESPJ.2010.2.1.2

Lewison, G., y Hartley, J. (2005). What's in a title? numbers of words and the presence of colons. Scientometrics, 63, 2, 341-356. https://doi.org/10.1007/ s11192-005-0216-0

Moattarian, A., y Alibabaee, A. (2015). Syntactic structures in research article titles from three different disciplines: applied linguistics, civil engineering, and dentistry. Journal of Teaching Language Skills, 34, 1, 27-50. https://dx.doi. org/10.22099/jtls.2015.3530

Morales, O. (2010). Los géneros escritos de la odontología hispanoamericana estruc- 
tura retórica y estrategias de atenuación en artículos de investigación, casos clínicos y articulos de revisión. Tesis doctoral, Barcelona, España: Universitat Pompeu Fabra. https://doi.org/10.13140/RG.2.2.22629.81123

Morales, O., Cassany, D., y Díaz, N. (2015). Resúmenes y abstracts de artículos científicos: variaciones retóricas e implicaciones didácticas. Acta Bioclínica, 5, 9, 33-46. Disponible en: http://erevistas.saber.ula.ve/index.php/actabioclini$\mathrm{ca} /$ article/view/5464/5269

Morales, O., Perdomo, B., Cassany, D., e Izarra, É. (2020a). Títulos de revisiones sistemáticas publicadas en español en revistas odontológicas. Panace@Revista de Medicina, Lenguaje y Traducción, xxi, 51, 62-74. Disponible en: https:// www.tremedica.org/wp-content/uploads/panacea20-51_00.pdf

Morales, O., Perdomo, B., Cassany, D., Tovar, R., e Izarra, É. (2020b). Linguistic structures and functions of thesis and dissertation titles in Dentistry. Lebende Sprachen, 65, 1, 1-25. https://doi.org/10.1515/les-2020-0003

Morales, O., Perdomo, B., Cassany, D., e Izarra, É., (2020c). Estructura retórica de tesis y trabajos de grado en Odontología: un estudio en Hispanoamérica y España. Íkala. Revista de Lenguaje y Cultura, 25, 2, 373-93. https://doi. org/10.17533/udea.ikala.v25n02a06

Morales, O., Perdomo, B., Cassany, D., e Izarra, É., (2020d). Estructura genérica de revisiones sistemáticas odontológicas publicadas en español. Círculo de Lingüistica Aplicada a la Comunicación, 83, 133-146. http://dx.doi.org/10.5209/ clac.70569

Nagano, R. (2015). Research article titles and disciplinary conventions: a corpus study of eight disciplines. Journal of Academic Writing, 5, 1, 133-144. https:// doi.org/10.18552/joaw.v5i1.168

Paiva, C., Lima, J., y Paiva, B. (2012). Articles with short titles describing the results are cited more often. Clinics, 67, 5, 509-513. Disponible en: http://www. scielo.br/pdf/clin/v67n5/a17v67n5.pdf

Rath, A. (2010a). Temporal function of research process nominal groups in the English titles. English for Specific Purposes World, 31, 10, 1-27.

Rath, A. (2010b). Dual function of first position nominal groups in research article titles: describing methods and structuring summary. Research in Applied Linguistics, 1, 2, 5-23.

Rath, A. (2010c). A Discourse analysis of first position nominal groups in research article titles. Taiwan International ESP Journal, 2, 1, 85-104.

Rincón, G., Narváez, E., y Roldán, C. (2011). Enseñar a comprender textos escritos en la universidad ¿qué y cómo se está haciendo? Lenguaje, 32, 183-211. Disponible en: http://revistalenguaje.univalle.edu.co/index.php/lenguaje/article/download/4810/6952

Sahragard, R., y Meihami, H. (2016). A diachronic study on the information provided by the research titles of applied linguistics journals. Scientometrics, 108, 
3, 1315-1331. https://doi.org/10.1007/s11192-016-2049-4

Salager-Meyer, F., y Alcaraz-Ariza, M. (2013). Medical case reports and titleology: A diachronic perspective (1840-2009). Revista de Lenguas para Fines Especificos, 19, 411-428. Disponible en: https://accedacris.ulpgc.es/bitstre am/10553/12247/1/0233536_00019_0018.pdf

Salager-Meyer, F., Alcaraz-Ariza, M., y Luzardo, M. (2013). Titling and authorship practices in medical case reports: a diachronic study. Communication \& Medicine, 10, 1, 63-80. https://doi.org/10.1558/cam.v10i1.63

Salager-Meyer, F., Alcaraz-Ariza, M., y Luzardo, M. (2017). Neutral, risky or provocative? Trends in titling practices in complementary and alternative medicine articles (1995-2016). Revista de Lenguas para Fines Específicos, 23, 2, 263-289. Disponible en: https://accedacris.ulpgc.es/bitstre am/10553/70379/2/0233536_00023_0022.pdf

Schulz, K., Altman, D., y Moher, D. (2010). CONSORT 2010 statement: updated guidelines for reporting parallel group randomised trials. BMC Medicine, 8, 1, 18-27. https://doi.org/10.1186/1741-7015-8-18

Shahidipour, V., y Alibabaee, A. (2017). Syntactic structures and rhetorical functions of electrical engineering, psychiatry, and linguistics research article titles in English and Persian: a cross-linguistic and cross-disciplinary study. Journal of Teaching Language Skills, 36, 1, 145-175. https://doi.org/10.22099/ jtls.2017.4066

Soler, V. (2007). Writing Titles in science: an exploratory study. English for Specific Purposes, 26, 90-102. https://doi.org/10.1016/j.esp.2006.08.001

Soler, V. (2009). Títulos científicos en lengua española: estudio exploratorio. Lebende Sprachen, 54, 2, 50-58. https://doi.org/10.1515/les.2009.017

Soler, V. (2011). Comparative and contrastive observations on scientific titles written in English and Spanish. English for Specific Purposes, 30, 2, 124-137. https://doi.org/10.1016/j.esp.2010.09.002

Soler, V. (2018). Estudio exploratorio de títulos de tesis doctorales redactados en lengua española. Lebende Sprachen, 63, 2, 374-392. https://doi.org/10.1515/ les-2018-0022

Swales, J. (1990). Genre analysis: English in academic research settings. Cambridge: Cambridge University Press.

Swales, J. (2004). Research genres: explorations and applications. Cambridge: Cambridge University Press.

Swales, J., y Feak, C. (2012). Academic writing for graduate students: essential tasks and skills. Ann Arbor, Michigan: University of Michigan Press.

Vathanalaoha, K., y Tangkiengsirisin, S. (2018). Genre analysis of experiment-based dental research article abstracts: Thai and international journals. 3 L: Language, Linguistics, Literature, 24, 3, 1-14. http://doi.org/10.17576/3L-2018-2403-01 Von Elm, E., Altman, D., Egger, M., Pocock, S., Gøtzsche, P., Vandenbroucke, J., 
y Strobe I. (2014). The Strengthening the Reporting of Observational Studies in Epidemiology (STROBE) Statement: guidelines for reporting observational studies. International Journal of Surgery, 12, 12, 1495-1499. https://doi. org/10.1016/j.ijsu.2014.07.013

Wang, Y., y Bai, Y. (2007). A corpus-based syntactic study of medical research article titles. System, 35, 3, 388-399. https://doi.org/10.1016/j.system.2007.01.005

Ye, Y. (2019). Macrostructures and rhetorical moves in energy engineering research articles written by Chinese expert writers. Journal of English for Academic Purposes, 38, 49-61. https://doi.org/10.1016/j.jeap.2019.01.007 